SUBJECT AREAS:

STRUCTURE OF SOLIDS

AND LIQUIDS

COMPUTATIONAL METHODS

THERMOELECTRICS

APPLIED PHYSICS

Received

1 September 2014

Accepted

4 December 2014

Published

22 January 2015

Correspondence and requests for materials should be addressed to B.L.H. (mebhuang@ ust.hk)

\title{
Thermal conductivity of skutterudite
} $\mathrm{CoSb}_{3}$ from first principles: Substitution and nanoengineering effects

\author{
Ruiqiang Guo', Xinjiang Wang' \& Baoling Huang ${ }^{1,2}$
}

\begin{abstract}
'Department of Mechanical and Aerospace Engineering, The Hong Kong University of Science and Technology, Clear Water Bay, Kowloon, Hong Kong, ${ }^{2}$ The Hong Kong University of Science and Technology Shenzhen Research Institute, Shenzhen, 518057 , China.
\end{abstract}

$\mathrm{CoSb}_{3}$-based skutterudites are promising intermediate-temperature thermoelectric materials and fundamental understanding of the thermal transport in $\mathrm{CoSb}_{3}$ is crucial for further improving its performance. We herein calculate the lattice thermal conductivity $\boldsymbol{\kappa}_{L}$ of $\mathrm{CoSb}_{3}$ with first-principles methods and conduct a comprehensive analysis on phonon mode contribution, relaxation time and mean free path (MFP) distributions. The contribution of optical phonons is found to be significant $(28 \%$ at $300 \mathrm{~K})$ and important optical modes usually involve two or more pnicogen atoms moving synchronously. The MFP $(\sim 135 \mathrm{~nm}$ at $300 \mathrm{~K})$ corresponding to $50 \% \kappa_{L}$ accumulation in $\mathrm{CoSb}_{3}$ is much larger than that predicted from the kinetic theory $(\sim 4 \mathrm{~nm})$, providing an opportunity to reduce $\boldsymbol{\kappa}_{L}$ by nanoengineering. The effects of elemental substitution and nanoengineering on $\boldsymbol{\kappa}_{L}$ are therefore investigated. A $10 \%$ substitution of Sb by As results in $57 \%$ reduction of $\boldsymbol{\kappa}_{L}$ while the in-plane (cross-plane) $\kappa_{L}$ of a $50-\mathrm{nm} \mathrm{CoSb}_{3}$ thin film is only $56 \%$ (33\%) of the bulk $\kappa_{L}$ at $300 \mathrm{~K}$. The impurity scattering and boundary scattering mainly suppress phonons in different frequency regimes. By combining these two effects, $\kappa_{L}$ can be reduced by more than $70 \%$ at $300 \mathrm{~K}$, potentially leading to much improved $Z T$ near room temperature.

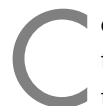

$\mathrm{OSb}_{3}$-based skutterudites are among the most prospective thermoelectric (TE) materials for intermediatetemperature power generation, owing to their high performance, low cost and great potential for tailoring the thermal and electrical transport properties through structural engineering. The efficiency of thermoelectric materials is often measured by a dimensionless figure of merit $Z T=S^{2} \sigma T /\left(\kappa_{L}+\kappa_{e}\right)$, where $S$ is the Seebeck coefficient, $\sigma$ the electrical conductivity, $T$ the temperature, and $\kappa_{L}$ and $\kappa_{e}$ are the lattice and electronic contributions to the thermal conductivity $k$, respectively. $\mathrm{CoSb}_{3}$ is a binary skutterudite, which has a general formula $\mathrm{MX}_{3}(I M 3)$ where $\mathrm{M}$ is a transition metal atom (Co, $\mathrm{Rh}$, or $\left.\mathrm{Ir}\right)$ and $\mathrm{X}$ is a pnicogen atom $(\mathrm{P}, \mathrm{As}$ or Sb) and is characterized by a cubic crystalline structure containing large cages and four-membered planar rings of $\mathrm{X}^{1}$. Binary skutterudites have superior $S^{2} \sigma$ due to their appropriate bandgaps and high carrier mobilities, but their thermal conductivities are also high, impeding their applications as efficient TE materials. A successful strategy to improve $Z T$ of skutterudites is through reducing $\kappa_{L}$ by filling atoms into lattice cages ${ }^{2-7}$, elemental substitution ${ }^{8-11}$ or nanoengineering ${ }^{11,12}$. Sale's et al. ${ }^{2}$ reported a $Z T$ of $\sim 1.0$ at $800 \mathrm{~K}$ in the filled skutterudite $\mathrm{LaFe}_{3} \mathrm{CoSb}_{12}$ that has a reduced $\kappa_{L}$ as low as $\sim 0.9 \mathrm{~W} / \mathrm{mK}$ due to the "rattling" atoms. Liu et al. ${ }^{9}$ and Su et al. ${ }^{10,11}$ synthesized n-type nanostructured $\mathrm{CoSb}_{2.75} \mathrm{Sn}_{0.05} \mathrm{Te}_{0.20}$ and $\mathrm{CoSb}_{2.75} \mathrm{Ge}_{0.05} \mathrm{Te}_{0.20}$ compounds in which the elemental substitution and in-situ generated quantum dots reduce $\kappa_{L}$ below $1.0 \mathrm{~W} / \mathrm{mK}$ at $\sim 800 \mathrm{~K}$, leading to $Z T s$ as high as 1.1. Qiu et al. ${ }^{13}$ achieved a maximum $Z T$ of $\sim 0.7$ at $600 \mathrm{~K}$ by doping $\mathrm{CoSb}_{3}$ with $\mathrm{Ga}$ atoms, which occupy both the voids and $\mathrm{Sb}$ sites. Toprak et al. ${ }^{12}$ obtained a highest $Z T \sim 0.17$ at $611 \mathrm{~K}$ for the pure nanocrystalline $\mathrm{CoSb}_{3}$ with average grain size of $220 \mathrm{~nm}$, which has a $\kappa_{L} \sim 0.8 \mathrm{~W} / \mathrm{mK}$ because of the enhanced boundary scattering. Although significant reduction of $\kappa_{L}$ has been achieved in these materials, the underlying mechanism is still not fully understood. For example, the average phonon mean free path (MFP) predicted from the kinetic theory is only $\sim 4 \mathrm{~nm}$, which cannot explain the substantially reduced $\kappa_{L}$ in the nanocrystalline $\mathrm{CoSb}_{3}$ with average grain size of a few hundred nanometers ${ }^{12}$. Also, the reported $\kappa_{L}$ values are still much larger than the theoretical alloy limit $\sim 0.3 \mathrm{~W} / \mathrm{mK}^{14}$. The open question is how $\kappa_{L}$ of skutterudites can be further reduced and by how much. The key to answer these questions is a rigorous prediction of $\kappa_{L}$ and a detailed knowledge of intrinsic phonon transport in $\mathrm{CoSb}_{3}$. To reduce $\kappa_{L}$ through structural engineering, phonon MFP distribution and spectral contributions to $\kappa_{L}$ is 
most important. Unfortunately, to date, accurate atomic-scale investigations on these details in $\mathrm{CoSb}_{3}$ are still rare.

Lattice dynamics in bulk $\mathrm{CoSb}_{3}$ have been intensively investigated. Lutz et al. ${ }^{15}$ and Nolas et al. ${ }^{16,17}$ investigated the lattice dynamics at the $\Gamma$ point using infrared and Raman spectroscopy, respectively. Rotter et al. ${ }^{18}$ measured the phonon dispersion along [001] direction by inelastic x-ray scattering experiments. First-principles calculations ${ }^{18-20}$ have been conducted to calculate the phonon dispersion and density of states (DOS), which show good agreements with the experimental results. A few classical force constant models have also been proposed by Lutz et al. ${ }^{15}$ and Feldman et al. ${ }^{21}$ through fitting with the first principles calculations and experimental phonon frequencies. These lattice dynamics investigations pave the way for the prediction of $\kappa_{L}$. Analytical phonon conductivity models ${ }^{1}$ based on Boltzmann transport equation (BTE), typically in Callaway-Holland form, have been developed to understand the effects of defects in bulk skutterudites. $\kappa_{L}$ of skutterudites can also be reasonably evaluated with Slack's model ${ }^{22}$. However, these models heavily rely on the fitting parameters and might blur the underlying heat transfer physics. Recently, Li et al. ${ }^{23}$ investigated the effects of fillers on $\kappa_{L}$ of fully-filled skutterudites by analyzing phonon relaxation times and phonon dispersion modifications obtained from the ab initio calculations. However, a comprehensive understanding of the mode-wise phonon transport in $\mathrm{CoSb}_{3}$ is still needed, which is useful for reducing $\kappa_{L}$ through selective doping or nanoengineering.

In this study, we accurately determine $\kappa_{L}$ of $\mathrm{CoSb}_{3}$ by using first principles and BTE. Detailed analysis on the mode-wise phonon relaxation time, MFP and spectral contributions to $\kappa_{L}$ has been conducted. It is found that optical phonons contribute significantly to $\kappa_{L}$ and the MFP $(\sim 135 \mathrm{~nm}$ at $300 \mathrm{~K})$ corresponding to $50 \% \kappa_{L}$ accumulation is surprisingly large, implying great potential for reducing $\kappa_{L}$ through nanoengineering. The effects of impurity scattering caused by element substitution and boundary scattering on spectral phonon transport are clarified. A microscopic understanding about the vibration behavior of the pnicogen rings and the effects of substitution is given. While the impurity scattering mainly affects optical phonons, the diffusive boundary can efficiently suppress acoustic phonon transport. It is shown that combining these two scattering mechanisms can significantly decrease $\kappa_{L}$. The improvement of $Z T$ benefited from $\kappa_{L}$ reduction may notably broaden the application temperature range of $\mathrm{CoSb}_{3}$-based skutterudites.

\section{Results}

$\kappa_{L}$ is determined by both the harmonic and anharmonic interatomic interactions and it is essential to understand the lattice dynamics in $\mathrm{CoSb}_{3}$ and the corresponding phonon distribution. The interaction strength between atoms from ab-initio calculations relies on the approximations to exchange and correlation, which will affect the lattice parameters and the forces on atoms used for extracting the phonon dispersion. We adopted the local density approximation (LDA) and generalized gradient approximation (GGA) treatments to optimize the $\mathrm{CoSb}_{3}$ structure, yielding a lattice constant of $8.92 \AA$ and $9.11 \AA$, respectively. Compared with the experimental value $a=$ $9.04 \AA^{24}$, the LDA underestimates $a$ by $1.3 \%$ while the GGA overestimates it by $1.1 \%$, agreeing well with previous ab-initio predictions ${ }^{18,20,25}$ and following the well-known overbinding and underbinding tendency of the LDA and GGA. Figures 1(a) and (b) show the phonon dispersions of bulk $\mathrm{CoSb}_{3}$ for LDA and GGA calculations along some high-symmetry directions, in comparison with the experimental results ${ }^{15-18}$. Overall both LDA and GGA reproduce well the acoustic branches while for optical phonons the LDA calculations show better agreements with the experimental values. Therefore, the LDA can be taken as a reliable base for the subsequent calculations. The acoustic group velocities are found to be $2601 \mathrm{~m} / \mathrm{s}$ for TA1 (transversal acoustic), $2881 \mathrm{~m} / \mathrm{s}$ for TA2 and $4716 \mathrm{~m} / \mathrm{s}$ for


Figure 1 Phonon dispersion along different high-symmetry paths for GGA (a) and LDA (b) calculations. Experimental results from Refs 15-18 are denoted by filled symbols. (c) Total and partial phonon density of states for $\mathrm{CoSb}_{3}$.

LA (longitudinal acoustic) respectively, agreeing with the experimental TA of $2643 \mathrm{~m} / \mathrm{s}$ and LA of $4590 \mathrm{~m} / \mathrm{s}^{26}$. It is known that the elastic constants are directly related to the acoustic group velocities. Our calculations yield the elastic constants $C_{11} 172.0 \mathrm{GPa}, C_{12}$ 43.7 $\mathrm{GPa}$ and $C_{44} 57.7 \mathrm{GPa}$, respectively, which are consistent with the experimental results ${ }^{27}$. Figure 1(c) shows the total phonon DOS of $\mathrm{CoSb}_{3}$ characterized by a large band gap between 6.3 and 7.4 THz. The band gap is related to the mass mismatches of constituent atoms. It has been shown that the band gap increases with increasing the cation/anion mass ratio in binary wurtzite nitrides ${ }^{28}$. The partial DOS of $\mathrm{CoSb}_{3}$ is also shown in Fig. 1(c) and significantly different vibration properties of $\mathrm{Co}$ and $\mathrm{Sb}$ atoms are illustrated. The vibration of Co atoms dominates the high frequency spectrum above $7.4 \mathrm{THz}$ while the low frequency spectrum below $6.3 \mathrm{THz}$ mainly comes from the vibration of $\mathrm{Sb}$ atoms. Such vibrational properties provide an opportunity to selectively tune the phonon spectrum in separate frequency regime by substituting different atoms in $\mathrm{CoSb}_{3}$.

$\kappa_{L}$ of bulk $\mathrm{CoSb}_{3}$ for LDA and GGA as a function of temperature are shown in Fig. 2, in comparison with the experimental results ${ }^{26,29}$. From $100 \mathrm{~K}$ to $900 \mathrm{~K}$, the LDA predictions are relatively larger than the GGA values and $\kappa_{L}$ shows an approximate $1 / T$ temperature dependence for both LDA and GGA, a common behavior for crystals. It is found that the LDA calculations match better with the experimental results while GGA tends to underestimate $\kappa_{L}$. For 


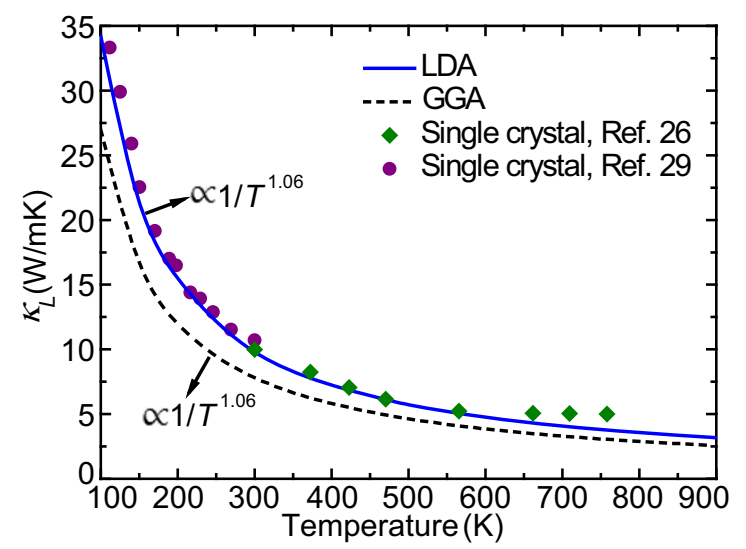

Figure $2 \mid \boldsymbol{\kappa}_{L}$ of $\mathrm{CoSb}_{3}$ for LDA and GGA with respect to temperature, compared with the experimental results (denoted by filled symbols) from Refs 26, 29.

example, the LDA $\kappa_{L}$ of $9.8 \mathrm{~W} / \mathrm{mK}$ at $300 \mathrm{~K}$ is very close to the experimental value $10 \mathrm{~W} / \mathrm{mK}$ of single crystals while the GGA produces a lower $\kappa_{L}$ of $7.8 \mathrm{~W} / \mathrm{mK}$. The latter agrees well with the result from Huang et al. ${ }^{20}$ obtained by using classical molecular dynamics simulations, in which the potentials were fitted from first-principles calculations with GGA. The underestimation by GGA calculations is mainly caused by the underbinding behavior of GGA, which makes the phonon dispersion shift to lower frequencies and results in slightly lower group velocities. The average acoustic group velocity for GGA is $\sim 8.7 \%$ smaller than that for LDA, which can lead to a thermal conductivity $\sim 17 \%$ lower according to the kinetic theory $\kappa_{L}=C_{v} V_{g} \lambda / 3=C_{v} V_{g}^{2} \tau / 3\left(C_{v}, V g, \lambda\right.$ and $\tau$ represent the phonon volumetric specific heat, group velocity, MFP and relaxation time, respectively). Above $700 \mathrm{~K}$, the calculated $\kappa_{L}$ are slightly lower than the experimental results, for which the electronic contributions (for example, $\sim 3 \%$ at $700 \mathrm{~K}$ based on Wiedemann-Franz law) to the total thermal conductivity have been removed. This deviation might be caused by bipolar heat conduction at high temperatures. The effects of phonon-isotope scattering on $\kappa_{L}$ is insignificant for $\mathrm{CoSb}_{3}$ in the temperature range considered. Natural isotopes result in a largest reduction $(4 \%)$ of $\kappa_{L}$ at $100 \mathrm{~K}$ with respect to the isotopically pure materials. Li et al. ${ }^{23}$ obtained a larger $\kappa_{L}$ of $11.5 \mathrm{~W} / \mathrm{mK}$ at $300 \mathrm{~K}$ using a similar method, which may be caused by the relatively small cutoff of $4 \AA$ adopted for the interaction range. Actually, the long-ranged interactions may be important for the phonon transport, which has been highlighted in previous investigations ${ }^{30}$. We found that the harmonic interatomic force constants almost vanish at a cutoff interatomic distance of $6.5 \AA$ (See the Supplementary Information).

The average MFP can be predicted according to the kinetic theory, yielding a very short average MFP of $\sim 4 \mathrm{~nm}$ at $300 \mathrm{~K}$. Considering the uneven contribution of different modes, this gray approximation might be misleading, as for MFP found in $\mathrm{Si}^{31}$. The knowledge of the contribution of different phonon modes to $\kappa_{L}$ is helpful for understanding the intrinsic phonon transport and can provide guidance for the structural engineering. Figure 3 shows the normalized $\kappa_{L}$ accumulation with respect to the MFP of bulk $\mathrm{CoSb}_{3}$ at 100,300 and $800 \mathrm{~K}$, respectively. The MFPs corresponding to $50 \% \kappa_{L}$ accumulation at 100, 300 and $800 \mathrm{~K}$ are respectively 595, 135 and $48 \mathrm{~nm}$, which, however, are surprisingly long. For example, the accumulation median MFP value for $\mathrm{CoSb}_{3}$ approaches $\sim 40 \%$ of the corresponding one of bulk $\mathrm{Si}(\sim 350 \mathrm{~nm})$ at $300 \mathrm{~K}$ although $\kappa_{L}$ of $\mathrm{Si}$ $\left(155 \mathrm{~W} / \mathrm{mK}\right.$ at $\left.300 \mathrm{~K}^{32}\right)$ is more than one order of magnitude higher. This difference can be simply explained by kinetic theory. When only acoustic phonons (contribute $\sim 70 \%$ to the overall $\kappa_{L}$ in $\mathrm{CoSb}_{3}$ at $300 \mathrm{~K})$ are considered, the thermal conductivity ratio $\kappa_{L}(\mathrm{Si}) /$ $\kappa_{L}\left(\mathrm{CoSb}_{3}\right) \approx\left[C_{v}(\mathrm{Si}) v_{g}(\mathrm{Si}) \lambda(\mathrm{Si})\right] /\left[C_{v}\left(\mathrm{CoSb}_{3}\right) v_{g}\left(\mathrm{CoSb}_{3}\right) \lambda\left(\mathrm{CoSb}_{3}\right)\right] \approx$

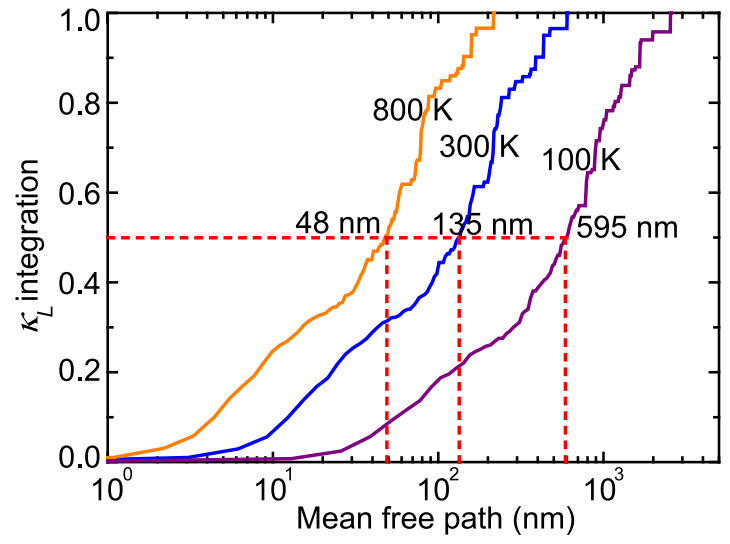

Figure $3 \mid$ Normalized $\kappa_{L}$ accumulation for $\mathrm{CoSb}_{3}$ at 100, 300 and $800 \mathrm{~K}$, as a function of phonon mean free path.

22.5, reasonably agreeing with the ab initio predictions. Here, the ratios of $C_{v}$ (estimated by the number density of unit cell through the Dulong-Petit law ${ }^{33}$ ), $v_{g}$ (that of $\mathrm{Si}$ is $\sim 6300 \mathrm{~m} / \mathrm{s}^{34}$ ) and $\lambda$ are about 4.5, 2 and 2.5, respectively. The relatively large phonon MFPs in $\mathrm{CoSb}_{3}$ rationalize the reported experimental results ${ }^{12}$ and suggest the potential to decrease $\kappa_{L}$ by nanoengineering. It is also noted that the phonon modes contribute unevenly to the thermal conductivity. For example, at $300 \mathrm{~K}$, the phonon modes with MFPs shorter than $100 \mathrm{~nm}$ contribute $42 \%$ to $\kappa_{L}$ while those with MFPs longer than $400 \mathrm{~nm}$ only contribute $10 \%$ to $\kappa_{L}$. Consequently, the gray approximation for MFP may lead to a large uncertainty in the analysis. This further confirms the necessity of using mode-dependent parameters for the modeling of $\kappa_{L}$. At $800 \mathrm{~K}$, the $50 \%$ thermal conductivity accumulation corresponds to a MFP of $48 \mathrm{~nm}$, which is relatively challenging to be further reduced by nanoengineering.

Figure 4(a) further shows the phonon scattering rates for the Umklapp and Normal processes with respect to frequency at $300 \mathrm{~K}$, which are obtained from the single-mode relaxation time approximation (SMRTA). The sum of the Normal and Umklapp scattering rates would be the inverse of the relaxation time, for which SMRTA and iterative method yield very close values and the deviation is less than $1 \%$. The scattering rates of three acoustic branches and optical modes are plotted separately. It can be found that for both processes the scattering rates generally increase with the increase of frequency, indicating the enhanced phonon scattering. Callaway ${ }^{35}$ suggested a $\omega^{2}$ dependence of scattering rates for the Umklapp process, which is often used to predict $\kappa_{L}$. Here, we found a quadratic dependence of the Umklapp scattering rates for acoustic phonons on frequency while Normal scattering rates are proportional to $\sim \omega^{0.5}$ at $300 \mathrm{~K}$. It is also noted that at low frequencies (typically $<1.5 \mathrm{THz}$ ) the Normal process dominates while the scattering rates of the two processes are comparable at higher frequencies (typically for optical modes). Due to different intrinsic scattering channels in materials, the frequency dependence of scattering rates may be different. For example, it has been reported that the acoustic scattering rates due to Normal and Umklapp processes at $277 \mathrm{~K}$ in bulk Si are proportional to $\omega^{2}$ and $\omega^{3}$ respectively ${ }^{31}$. Figure $4(\mathrm{~b})$ shows the mode-averaged MFP distributions with respect to frequency at 100,300 and $800 \mathrm{~K}$, respectively. Typically, the MFP decreases with the increase of temperature due to the enhanced phonon scattering. Because of the reduction of the relaxation time and group velocity, the MFP will be suppressed when the frequency increases. From Fig. 4(b), one can find that acoustic phonons generally possess a relatively long MFP. At $300 \mathrm{~K}$, the MFPs of acoustic phonons increase from $20 \mathrm{~nm}$ to $\sim 500 \mathrm{~nm}$ below $2.2 \mathrm{THz}$ while most optical phonons $(f>2.2 \mathrm{THz})$ possess a MFP shorter than $10 \mathrm{~nm}$. Therefore, nanoengineering will significantly suppress acoustic phonons while optical phonons will be less affected. Even at $800 \mathrm{~K}$, the MFPs of most acoustic phonons 

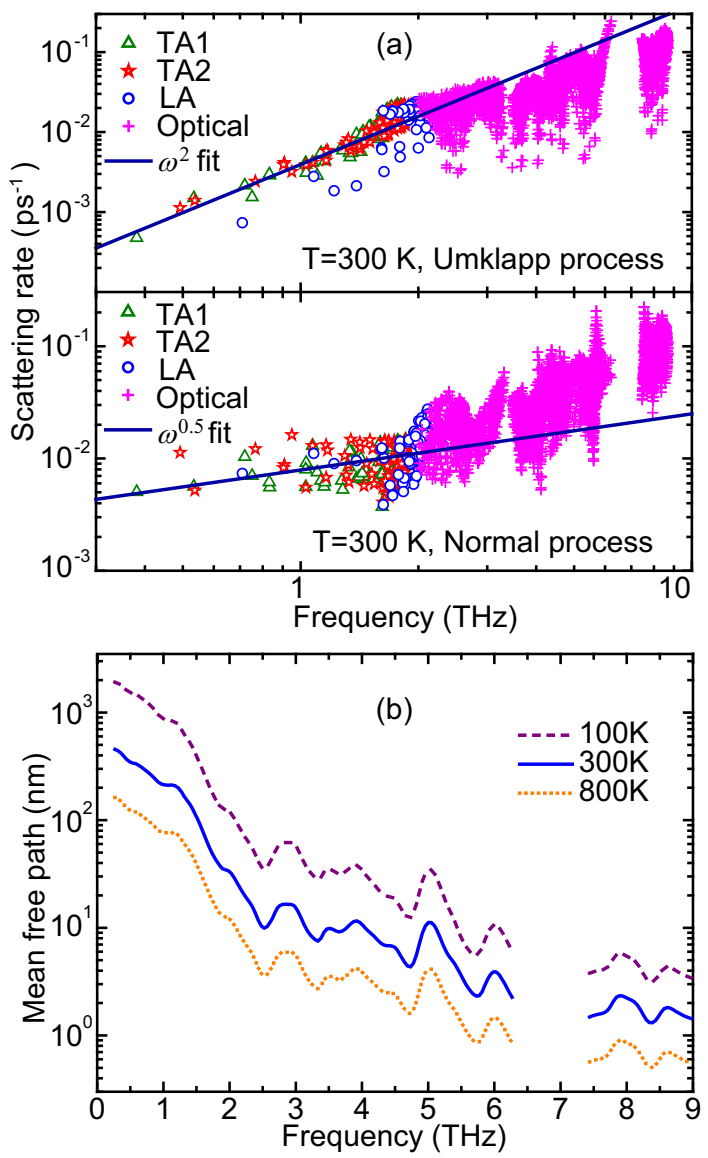

Figure $4 \mid$ Phonon scattering rates for Umklapp process and Normal process at $300 \mathrm{~K}(\mathrm{a})$ and average mean free path (b) at 100,300 and $800 \mathrm{~K}$ with respect to frequency for $\mathrm{CoSb}_{3}$.

are above tens of nanometers while optical phonons typically have a MFP shorter than $5 \mathrm{~nm}$.

Figure 5(a) shows $\kappa_{L}$ contribution with respect to frequency at 100, 300 and $800 \mathrm{~K}$, respectively. The curves for different temperatures have a similar shape with a peak position at $\sim 1.2 \mathrm{THz}$. The acoustic phonons between 0.5 and $2.0 \mathrm{THz}$ dominate $\kappa_{L}$ contribution. Despite the dominance of acoustic phonons, the optical phonons still contribute $\sim 27.7 \%$ of the total $\kappa_{L}$ at $300 \mathrm{~K}$. At $800 \mathrm{~K}$, the relative contribution of optical phonons slightly increases to $28.3 \%$. However, the optical phonons with frequency $>5.5 \mathrm{THz}$ contribute little to $\kappa_{L}$ at all these temperatures. Significance of optical phonons has also been highlighted in some previous works ${ }^{36-38}$. In materials such as $\mathrm{Si}$ nanowires ${ }^{37}, \mathrm{PbSe}^{38}$ and $\mathrm{PbTe}^{38}$, optical phonons contribute $\sim 20 \%$ to $\kappa_{L}$. One can find that there are three peaks P1, P2 and P3 in the optical frequency regime, which result from some important phonon modes with relative large $\kappa_{L}$ contributions. The corresponding vibration modes are shown in the bottom panel of Fig. 5(a). The skutterudites contain two typical substructures: the pnicogen ring ( $\mathrm{Sb} 4$ ring for $\mathrm{CoSb}_{3}$ ) and the octahedron, as shown in the inset of Fig. 5(a). It is of great importance to observe the vibration of the pnicogen rings because dominant heat-carrying modes in skutterudites are related with their vibrations ${ }^{8,10,11}$. Due to the covalent characteristic, the bonds of Sb4 rings are relatively strong. The force constants obtained from first principles also support this (Fig. 6). The vibration modes are shown in a typical primitive cell, where the middle Co atom is surrounded by 6 first-neighbor $\mathrm{Sb}$ atoms that form an octahedron. Based on the space symmetry, one can find that the diagonal four Sb atoms 9, 10, 11 and 16 would form a Sb4 ring, as shown in the inset of Fig 5(a). It is found that the diagonal atoms on the Sb4 ring have parallel eigenvectors but may vibrate out of sync.
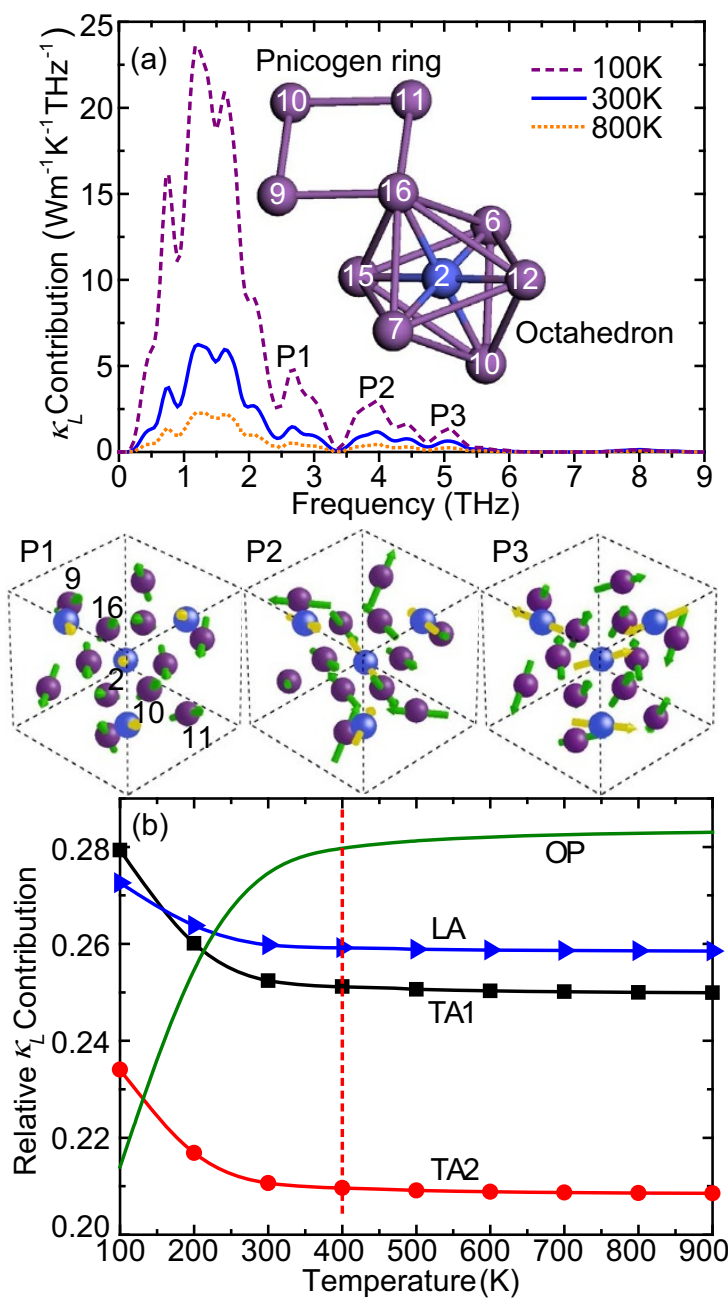

Figure $5 \mid$ (a) $\kappa_{L}$ contribution with respect to frequency at 100, 300 and $800 \mathrm{~K}$ for $\mathrm{CoSb}_{3}$. The optical vibration modes corresponding to the peaks P1, P2 and P3 are shown in the bottom (eigenvectors are indicated by arrows). Inset shows a typical skutterudite structure consisting of the pnicogen ring and octahedron. Co and $\mathrm{Sb}$ atoms are colored by blue and purple, respectively. (b) Relative $\kappa_{L}$ contribution of TA1, TA2, LA and optical (OP) phonon branches with respect to temperature.

For the mode P1, the rings exhibit a translational-like motion with all ring atoms vibrate almost synchronously. For the mode P2, the atoms on each long $\mathrm{Sb}-\mathrm{Sb}$ bond move together with the bond length slightly changed during the vibration while the two long bonds (16-9 and 11-10) vibrate in the opposite direction. The mode P3 has a similar vibration behavior for the $\mathrm{Sb} 4$ ring except that the atoms on the short $\mathrm{Sb}-\mathrm{Sb}$ bonds are bonded and move together. Generally, the six Sb atoms in the octahedron vibrate asynchronously and along different directions, which leads to significant deformation for the octahedron. From these typical modes, it can be found that the atoms on the pnicogen rings seldom move randomly; instead, two or more pnicogen atoms are usually bonded together, which can be attributed to their strong covalent bonds. Therefore, if this bonding behavior is disturbed $\kappa_{L}$ contribution of related phonon modes may be significantly reduced. This provides a microscopic understanding about $\kappa_{L}$ suppression caused by the substitution of pnicogen atoms $s^{8,10,11}$. Specifically, Chi et al. ${ }^{8}$ found that double substitution is an effective way to disturb the pnicogen rings.

It is informative to examine $\kappa_{L}$ contribution from different phonon branches, as shown in Fig. 5(b). The contribution of optical phonons is plotted by summing up over all optical phonon modes. Due to the enhanced Umklapp scattering, the contribution of acous- 

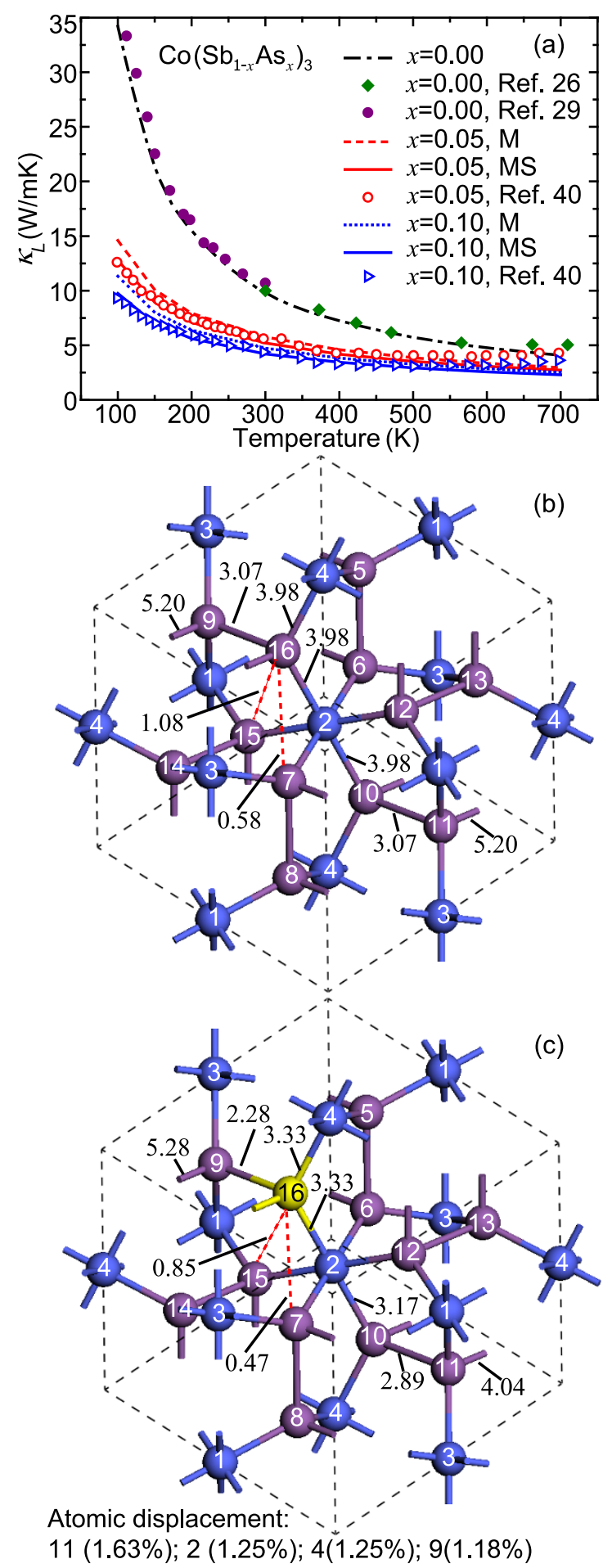

Figure $6 \mid$ (a) Temperature-dependent $\kappa_{L}$ of $\mathrm{Co}\left(\mathrm{Sb}_{1-x} \mathrm{As}_{x}\right)_{3}$. The ab-initio and experimental results from Refs 26, 29 (single crystal) and 40 (polycrystal) are represented by lines and symbols, respectively. " $\mathrm{M}$ " means only the mass variance is considered while "MS" incorporates the effects of both mass variance and strain field fluctuation. Comparisons between the longitudinal force constants of $\mathrm{Co}_{4} \mathrm{Sb}_{12}$ (b) and $\mathrm{Co}_{4} \mathrm{Sb}_{11} \mathrm{As}_{1}$ (c) obtained from first principles are also shown. Co atoms 1-4 and $\mathrm{Sb}$ atoms $5-16$ are colored by blue and purple, respectively. The Sb atom 16 is substituted by As and represented by yellow color.

tic phonons decreases as the temperature increases. The contributions from the three acoustic branches are comparable and the longitudinal branch contributes the most above $160 \mathrm{~K}$, probably due to its large group velocity. When temperature increases, owing to the fast reduction in the contribution of acoustic phonons, the relative contributions from optical phonons increase although their absolute contributions to $\kappa_{L}$ decrease because of the enhanced scattering, as shown in Fig. 5(a). Overall, the relative contributions of different branches vary little with temperature and almost keep constant above $400 \mathrm{~K}$.

The phonon contribution analysis points out the possible directions to tune $\kappa_{L}$ of $\mathrm{CoSb}_{3}$. Considering the relative large MFPs of acoustic phonons, nanoengineering will be effective in reducing $\kappa_{L}$ by boundary scattering. Also, the effect of impurity scattering on $\kappa_{L}$ should be notable because of the significant contribution from optical phonons. Thus, it is expected that a combination of these two mechanisms will greatly suppress $\kappa_{L}$ of $\mathrm{CoSb}_{3}$. Next, we'll examine how much $\kappa_{L}$ can be reduced by these two scatterings and analyze the underlying mechanisms. Impurity scattering can be generated in $\mathrm{CoSb}_{3}$ by two approaches: filling atoms into the lattice cages and elemental substitution. Filled $\mathrm{CoSb}_{3}$ structures have been extensively studied $^{2,20,39}$. In fact, there are more elements available for the substitution of Co and Sb elements, and most processes can be used for the synthesis. According to the DOS, it is known that $\mathrm{Sb}$ atoms dominate the low-frequency phonon spectrum. More importantly, the substitution of the $\mathrm{Sb}$ atom will significantly disturb the pnicogen rings, which play an important pole in the thermal transport of skutterudites. Therefore, we herein introduced impurity scattering by substituting $\mathrm{Sb}$ atoms with As atoms.

$\kappa_{L}$ of $\mathrm{Co}\left(\mathrm{Sb}_{1-x} \mathrm{As}_{x}\right)_{3}$ with different As concentrations as a function of temperature were calculated by including both effects of mass variance and strain field fluctuation, in comparison with those calculated by only considering mass variance and experimental results $^{26,29,40}$, as shown in Fig. 6(a). It is found that the calculated results for both cases show a similar tendency to the experimental values. The substitution of $\mathrm{Sb}$ can significantly lower $\kappa_{L}$ of $\mathrm{CoSb}_{3}$, e.g., a $10 \%$ substitution of $\mathrm{Sb}$ element with As results in a $57.1 \%$ reduction of $\kappa_{L}$. The further reduction of $\kappa_{L}$ caused by strain field scattering is significant below $200 \mathrm{~K}$ but gradually decreases as the temperature increases. This is because the relative contribution of strain filed scattering becomes smaller due to the enhanced phonon scattering at higher temperatures. Our calculations indicate that the scattering due to mass variance is much larger than strain field scattering, which is different from the previous estimations based on adjustable parameters ${ }^{40}$. It is also noted that the experimental results are higher than the calculated values above $600 \mathrm{~K}$. The electronic contribution is found to be less than $4 \%$ to the total thermal conductivity, implying that bipolar heat conduction may be important at high temperatures. The strain field scattering originates from the modification of atom position and interatomic coupling force $e^{40,41}$. To confirm this, we calculated the atomic displacements and the longitudinal force constants $\Gamma_{\mathrm{L}}$ between various atoms in the substituted and un-substituted structures, as shown in Figs. 6(b) and (c). It is found that the atomic displacements for the four neighbor atoms of As $(>1.1 \%)$ are significantly larger than those of other atoms $(<0.6 \%)$. In $\mathrm{CoSb}_{3}$, the largest three $\Gamma_{\mathrm{L}}$ come from the Co-Sb bond $\left(3.98 \mathrm{eV} / \AA^{2}, 16-4\right)$ in the octahedron and the short $\left(5.20 \mathrm{eV} / \AA^{2}, 16-\right.$ 11) and long (3.07 eV/ $\left.\AA^{2}, 16-9\right) \mathrm{Sb}-\mathrm{Sb}$ bonds in the Sb4 ring. The substitution of $\mathrm{Sb}$ atom 16 by As atom has a significant affection on the surrounding bonds. Most $\Gamma_{\mathrm{L}}$ between the atom 16 and other atoms are reduced, indicating that the bonds are softened. For example, the $\Gamma_{\mathrm{L}} 16-9,16-11$ and $16-4$ are reduced by $25.8 \%, 22.4 \%$ and $16.3 \%$ respectively. Similar bond softening was also reported in other studies on skutterudites with filled atoms ${ }^{20}$.

To investigate the effect of boundary scattering on thermal conductivity of $\left.\mathrm{Co}\left(\mathrm{Sb}_{1-x} \mathrm{As}\right)_{x}\right)_{3}$, we obtained the thickness dependence of the in-plane and cross-plane $\kappa_{L}$ of $\mathrm{Co}\left(\mathrm{Sb}_{1-x} \mathrm{As}_{x}\right)_{3}$ films at $300 \mathrm{~K}$, as shown in Fig. 7. For both structures $(x=0.0$ and 0.1$)$, boundary scattering begins to markedly affect $\kappa_{L}$ when the thickness is smaller 


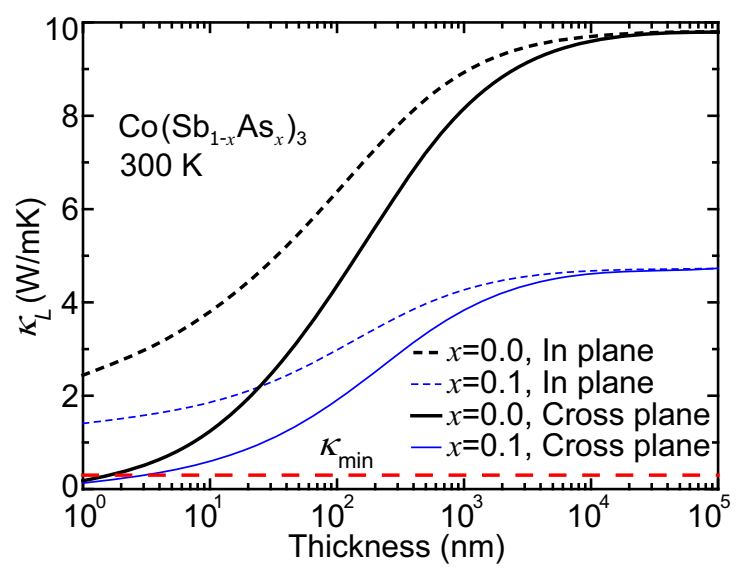

Figure $7 \mid$ Thickness dependence of $\kappa_{L}$ in $\operatorname{Co}\left(\mathrm{Sb}_{1-x} A s_{x}\right)_{3}$ for $x=0.0$ and 0.1. The theoretical minimum $\kappa_{\min }$ is also shown.

than $\sim 1 \mu \mathrm{m}$. If the thickness of un-substituted $\mathrm{CoSb}_{3}$ decreases to $50 \mathrm{~nm}$, its in-plane (cross-plane) $\kappa_{L}$ can be reduced by $44.1 \%$ (67.4\%). It is expected that boundary scattering will have a stronger influence on $\kappa_{L}$ of the substituted structures, in which the contribution of low-frequency phonons should be relatively larger due to the impurity scattering. For $\mathrm{Co}\left(\mathrm{Sb}_{0.9} \mathrm{As}_{0.1}\right)_{3}$ thin film with a thickness of $50 \mathrm{~nm}$, its in-plane (cross-plane) $\kappa_{L}$ is reduced by $73.9 \%(85.9 \%)$ with respect to the bulk value of $\mathrm{CoSb}_{3}$. The thickness corresponding to $50 \%$ reduction of in-plane $\kappa_{L}$ in the substituted one is $34 \mathrm{~nm}$. It is inspiring to find that combining these two scatterings can result in a greatly reduced $\kappa_{L}$. For example, the in-plane (cross-plane) $\kappa_{L}$ of $\mathrm{Co}\left(\mathrm{Sb}_{0.9} \mathrm{As}_{0.1}\right)_{3}$ with a thickness of $50 \mathrm{~nm}$ is only $2.6(1.4) \mathrm{W} / \mathrm{mK}$ at $300 \mathrm{~K}$, much lower than $9.8 \mathrm{~W} / \mathrm{mK}$ for bulk $\mathrm{CoSb}_{3}$. It has been experimentally found that the power factor of bulk $\mathrm{Co}\left(\mathrm{Sb}_{0.9} \mathrm{As}_{0.1}\right)_{3}$ increases by $176 \%$ with respect to that of the $\mathrm{CoSb}_{3}{ }^{40}$. Considering the short electronic MFP, it is reasonable to assume a little-deteriorated power factor in corresponding thin films. As a result, $Z T$ of bulk $\mathrm{CoSb}_{3}$ can be potentially improved by about 10 (19) times at room temperature.

\section{Discussion}

To further understand the influence of impurity scattering and boundary scattering, we calculated $\kappa_{L}$ contribution with respect to frequency and MFP distribution for different structures, as shown in Figs. 8(a) and (b). From Fig. 8(a), one can find that the substitution mainly affects the phonons with frequency $>1 \mathrm{THz}$, the contributions of which to $\kappa_{L}$ are greatly reduced. But acoustic phonons with frequency $<1 \mathrm{THz}$ are almost unaffected. The reduction of film thickness also has an impact on both acoustic and optical phonons. When the thickness of $\mathrm{CoSb}_{3}$ reduces to $50 \mathrm{~nm}$, the in-plane contributions of acoustic and optical phonons are reduced by $54.2 \%$ and $14.7 \%$ respectively. For the $\mathrm{Co}\left(\mathrm{Sb}_{0.9} \mathrm{As}_{0.1}\right)_{3}$ thin film with the same thickness, compared with its bulk, the boundary scattering reduces the in-plane contributions of acoustic and optical phonons by $57.5 \%$ and $42.3 \%$ respectively. For both thin films, the further reduction of $\kappa_{L}$ caused by boundary scattering is mainly due to the suppression of low-frequency phonons. Figure 8 (b) shows the MFP distribution with respect to phonon frequency at $300 \mathrm{~K}$. It is noted that the phonon MFPs between 1 and $6 \mathrm{THz}$ are mainly decreased due to the substitution. When the substitution fraction increases, the MFPs of these phonons are further reduced. But the MFPs of acoustic phonons with frequency $<1 \mathrm{THz}$ are hardly affected. The modification of MFP caused by boundary scattering is very different. For the $\mathrm{CoSb}_{3}$ thin film with a thickness of $50 \mathrm{~nm}$, the MFPs of most acoustic phonons are reduced by more than $50 \%$ while the MFPs of highfrequency phonons $(>3 \mathrm{THz})$ almost remain the same. For the $\mathrm{Co}\left(\mathrm{Sb}_{0.9} \mathrm{As}_{0.1}\right)_{3}$ thin film, however, the MFPs of optical phonons
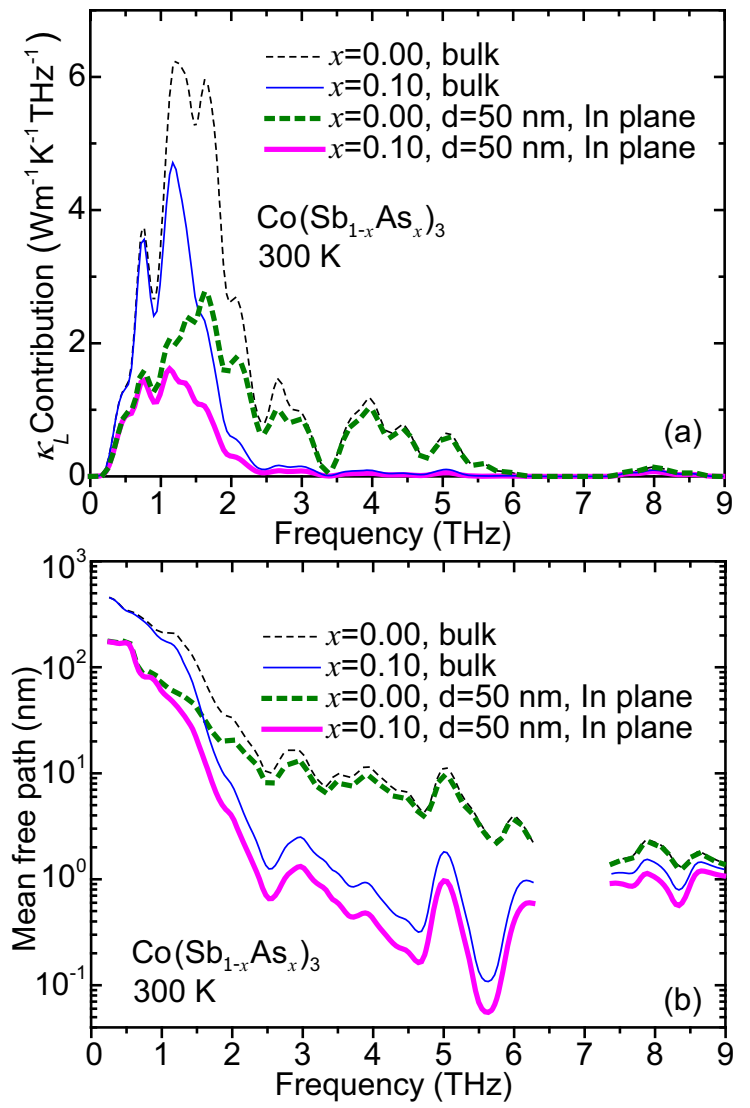

Figure $8 \mid \kappa_{L}$ contribution (a) and phonon mean free path (b) with respect to frequency for bulk $\mathrm{Co}\left(\mathrm{Sb}_{1-X} \mathrm{As}_{X}\right)_{3}$ and thin films of a thickness of $50 \mathrm{~nm}$ with different As concentrations.

from 2.2 to $6 \mathrm{THz}$ are also remarkably decreased by $\sim 50 \%$ with respect to its bulk, accounting for the corresponding significant reduction of $\kappa_{L}$.

In summary, the intrinsic $\kappa_{L}$ of $\mathrm{CoSb}_{3}$ has been accurately predicted using BTE with the harmonic and anharmonic parameters obtained from first-principles calculations. A comprehensive analysis about phonon mode contribution, relaxation time and MFP distributions was conducted. Optical phonons contribute significantly to $\kappa_{L}(28 \%$ at $300 \mathrm{~K})$ and their relative contributions slightly increase with temperature. To reduce $\kappa_{L}$, the suppression of optical phonons should be considered. We also found that the MFP $(\sim 135 \mathrm{~nm}$ at $300 \mathrm{~K})$ corresponding to $50 \% \kappa_{L}$ accumulation in $\mathrm{CoSb}_{3}$ is much larger than that predicted from the kinetic theory $(\sim 4 \mathrm{~nm})$, providing an opportunity to reduce $\kappa_{L}$ by nanoengineering. Interestingly, the vibrations of some important optical modes show that two or more pnicogen ring atoms usually move synchronously due to their strong covalent bonds. This provides a microscopic understanding about $\kappa_{L}$ reduction caused by the substitution of pnicogen atoms.

Accordingly, we investigated the effects of impurity scattering generated by elemental substitution and boundary scattering in thin films on $\kappa_{L}$. To accurately predict $\kappa_{L}$ of the substituted structures, both mass variance and strain field fluctuation should be considered due to the significant atomic displacements and bond softening. It is found that a $10 \%$ substitution of $\mathrm{Sb}$ element with As results in a $57.1 \%$ reduction of $\kappa_{L}$ while the in-plane (cross-plane) $\kappa_{L}$ of a $50 \mathrm{~nm} \mathrm{CoSb} b_{3}$ thin film is only $55.9 \%$ (32.6\%) of the bulk value at $300 \mathrm{~K}$. By combining these two effects, $\kappa_{L}$ can be reduced by $73.9 \%$ (85.9\%). Further analysis shows that the substitution mainly suppresses the phonons with frequency $>1 \mathrm{THz}$. In thin films of $\mathrm{Co}\left(\mathrm{Sb}_{1-x} \mathrm{As}_{x}\right)_{3}$, the reduction of $\kappa_{L}$ is mainly caused by the great 
reduction in MFPs of acoustic phonons. In this work, we demonstrate that elemental substitution combined with boundary scattering is an effective approach for reducing $\kappa_{L}$ of $\mathrm{CoSb}_{3}$. The calculated phonon transport details provide guidance for the further reduction of $\kappa_{L}$, which can be achieved if multiple scattering mechanisms are reasonably manipulated. The enhancement of $Z T$ benefited from the substantially reduced $\kappa_{L}$ may significantly enlarge the efficient working temperature range of $\mathrm{CoSb}_{3}$-based thermoelectrics.

\section{Methods}

The lattice thermal conductivity can be predicted based on phonon BTE with harmonic and anharmonic interatomic force constants (IFCs) as inputs, which are determined from ab initio calculations. Recently, many other materials $\mathrm{s}^{31,38,42-46}$ have been successfully predicted by this method, which show good agreements with the experimental results.

BTE. At equilibrium state, the phonon distribution is determined by the BoseEinstein distribution function $n_{\xi}^{0}$, where $\xi(q, j)$ represents a phonon mode denoted by the wavevector $q$ and the phonon dispersion branch $j$. By applying a small temperature gradient $\nabla T$ to disturb the phonon distribution function $n_{\xi}=n_{\xi}^{0}+n_{\xi}^{1}$, the BTE can be written as

$$
v_{g}(\xi) \cdot \nabla T \frac{\partial n_{\xi}^{0}}{\partial T}=\left.\frac{\partial n}{\partial t}\right|_{s c a t t},
$$

where the nonequilibrium part $n_{\xi}^{1}$ is proportional to the small $\nabla T$ and $\mathrm{v}_{g}(\xi)$ is the group velocity. The left-hand side represents phonon diffusion induced by the temperature gradient and the right-hand side represents the phonon scattering rate due to all scattering process. Matthiessen's rule is often adopted to sum up the effects of independent multiple scattering mechanisms. This study only considers three major scattering mechanisms in $\mathrm{CoSb}_{3}$, i.e., intrinsic phonon-phonon scattering, impurity scattering and boundary scattering.

The phonon-phonon scattering relaxation time $\tau_{p-p}$ can be calculated by anharmonic lattice dynamics calculations. We herein only consider the three-phonon scattering process that is dominant below $1000 \mathrm{~K}$ although it is possible to consider the fourth-order and even higher order anharmonic effects in the calculations, which might play a role at very high temperatures. Three-phonon scattering rates computed from Fermi's golden rule is related to the third-order anharmonic interactions ${ }^{42}$ through

$$
P_{\xi^{\prime}, \xi^{\prime}, \xi^{\prime \prime}}^{ \pm}=\frac{\hbar \pi}{4 N_{0}}\left(n_{\xi}^{0}+1\right)\left(n_{\xi^{\prime}}^{0}+\frac{1}{2} \pm \frac{1}{2}\right) n_{\xi^{\prime \prime}}^{0}\left|V_{\xi^{\prime}, \xi^{\prime}, \xi^{\prime \prime}}^{ \pm}\right|^{2} \frac{\delta\left(\omega(\xi) \pm \omega\left(\xi^{\prime}\right)-\omega\left(\xi^{\prime \prime}\right)\right)}{\omega(\xi) \omega\left(\xi^{\prime}\right) \omega\left(\xi^{\prime \prime}\right)}
$$

where $\omega$ is phonon frequency. In this process, the conservation of energy and momentum should be satisfied, i.e., $\omega(\xi) \pm \omega\left(\xi^{\prime}\right)=\omega\left(\xi^{\prime \prime}\right)$ and $q^{\prime \prime}+q+q^{\prime}=G$, where $G$ is either 0 for the Normal process or a nonzero reciprocal lattice vector for the Umklapp process. The interaction strength $V_{\xi_{\xi} \xi^{\prime}, \xi^{\prime \prime}}^{ \pm}$among three phonon eigenmodes $\xi, \xi^{\prime}$ and $\xi^{\prime \prime}$ can be determined by

$$
V_{\xi, \xi^{\prime}, \xi^{\prime \prime}}^{ \pm}=\sum_{b, l^{\prime} b^{\prime}, l^{\prime \prime} b^{\prime \prime}} \sum_{\alpha, \beta, \gamma}\left[\begin{array}{l}
\Psi_{\alpha \beta \gamma}\left(0 b, l^{\prime} b^{\prime}, l^{\prime \prime} b^{\prime \prime}\right) e^{i\left(q^{\prime} l^{\prime}+q^{\prime \prime} l^{\prime \prime}\right)} \\
\times \frac{\mathbf{e}_{\alpha}(b \mid \xi)}{\sqrt{m_{b}}} \frac{\mathbf{e}_{\beta}\left(b^{\prime} \mid \pm \xi^{\prime}\right)}{\sqrt{m_{b^{\prime}}}} \frac{\mathbf{e}_{\gamma}\left(b^{\prime \prime} \mid-\xi^{\prime \prime}\right)}{\sqrt{m_{b^{\prime}}}}
\end{array}\right],
$$

where $\psi_{\alpha \beta \gamma \gamma}\left(0 b, l^{\prime} b^{\prime}, / " b\right.$ ") represents the third-order force constants in terms of atoms $b, b^{\prime}$ and $b^{\prime \prime}$ in lattice $0, l^{\prime}$ and $l^{\prime \prime}$ of a crystal consisting of $N_{0}$ cell.

The isotopic impurity scattering rate can be calculated by ${ }^{47}$

$$
P_{\xi^{\prime}}^{p-i}=\frac{\pi g(b) \omega(\xi) \omega\left(\xi^{\prime}\right)}{2} n_{\xi}^{0}\left(n_{\xi^{\prime}}^{0}+1\right) \sum_{b, \alpha}\left|\mathbf{e}_{\alpha}^{*}(b \mid \xi) \mathbf{e}_{\alpha}\left(b \mid \xi^{\prime}\right)\right|^{2} \delta\left(\omega(\xi)-\omega\left(\xi^{\prime}\right)\right),
$$

where the mass variance is often characterized as an average parameter

$$
g(b)=\sum_{s} f_{s}(b)\left[1-M_{s}(b) / \bar{M}(b)\right]^{2},
$$

where $f_{s}(b)$ and $M_{s}(b)$ are the concentration and mass of the sth isotope of atom $b$, respectively, $\bar{M}(b)$ is the average mass of the $b$ th atom in the unit cell. Naturally, Co is composed of one stable isotope ${ }^{59} \mathrm{Co}$ while $\mathrm{Sb}$ consists of ${ }^{121} \mathrm{Sb}(57.36 \%)$ and ${ }^{123} \mathrm{Sb}$ $(42.64 \%)$.
For the case of substitution, the substitutional atom differs from the host atom not only in its mass, but also in its size and the coupling force to its neighbor atoms, which will result in the strain field scattering. Instead of $g(b)$, a convenient scattering parameter $a_{s}$, including both the effects of mass variance and strain field fluctuation, is defined as ${ }^{8,41}$

$$
a_{s}=x(1-x)\left[\left(\frac{\Delta M}{M}\right)^{2}+3 \gamma_{G}^{2}\left(\frac{\Delta R}{R}\right)^{2}\right],
$$

where $x$ is the atomic fraction of the impurity atoms, $\Delta M$ and $\Delta R$ are the atomic mass and radius difference between the impurity and host atoms, $\gamma_{\mathrm{G}}$ is the Grüneisen constant, and $M$ and $R$ are the average atomic mass and radius. The first term about mass corresponds to the mass variance in Eq. (5). The mode Grüneisen constants $\gamma_{i}$ which provide an estimate of the anharmonicity of the bonds, were directly calculated from first principles. An average $\gamma_{\mathrm{G}}$ was obtained for phonons contributing to the thermal transport according to $\gamma_{G}=\sum_{i} \gamma_{i} \mathrm{C}_{v, i} / \sum_{i} \mathrm{C}_{v, i}{ }^{48}$.

Assuming the coordinate $z$ is along the cross-plane direction of thin films, the boundary scattering rate can be quantified as

$$
\frac{1}{P_{p-b}}=\frac{1+p}{1-p} \frac{L}{2\left|v_{z}\right|}
$$

where $L / 2\left|V_{z}\right|$ represents the average time duration for a phonon to transport from one boundary to the other, provided evenly distributed possibility of emergent angle after scattering. The specularity parameter $p$, ranging from 0 to 1 , is used to account for the possibility of specular reflection on the surface. In this work, $p$ is set to 0 , i.e., a complete diffusive scattering on the boundary is assumed. Therefore, the calculations will lead to a lower bound of $\kappa_{L}$ for thin films. For the calculations of in-plane and cross-plane thermal conductivity, the $x$ and $z$ components of group velocity are adopted respectively.

Equation (1) is solved using an iterative approach ${ }^{49-51}$ to obtain the relaxation time $\tau(\xi)$, which is used to calculate the lattice thermal conductivity

$$
\kappa_{L, \alpha \beta}=\sum_{\xi} C_{v}(\xi) v_{g, \alpha}(\xi) v_{g, \beta}(\xi) \tau(\xi)
$$

where $\kappa_{L}$ is a second-order tensor with the subscripts $\alpha$ and $\beta$ denoting its components. $C_{v}$ is related to the mode frequency by

$$
C_{v}(\xi)=\frac{k_{B}}{V}\left[\frac{\hbar \omega(\xi)}{k_{B} T}\right]^{2} \frac{\exp \left(\hbar \omega(\xi) / k_{B} T\right)}{\left[\exp \left(\hbar \omega(\xi) / k_{B} T\right)-1\right]^{2}},
$$

where $k_{B}$ is the Boltzmann constant and $V$ is the volume of the unit cell. $C_{v}$ and $v_{g}=$ $\partial \omega / \partial q$ can be determined by the phonon dispersion relations obtained from harmonic lattice dynamics calculations ${ }^{52}$. To analyze the scatterings caused by the Normal and Umklapp processes, we also calculated $\tau(\xi)$ by adopting the single-mode relaxation time approximation. The relaxation times obtained in these two different methods are very close and the deviation is less than $1 \%$.

The phonon dispersion relations of a crystal can be obtained by solving the eigenvalue problem through diagonalizing the Fourier-transformed harmonic interatomic force constants (dynamical matrix) ${ }^{33}$, as shown in equations (10) and (11)

$$
\sum_{b^{\prime} \beta} D_{\alpha \beta}\left(b b^{\prime} \mid q\right) \mathbf{e}_{\beta}\left(b^{\prime} \mid \xi\right)=\omega^{2}(\xi) \mathbf{e}_{\alpha}(b \mid \xi)
$$

$$
D_{\alpha \beta}\left(b b^{\prime} \mid q\right)=\frac{1}{\sqrt{m_{b} m_{b^{\prime}}}} \sum_{l^{\prime}} \Phi_{\alpha \beta}\left(0 b, l^{\prime} b^{\prime}\right) e^{i q l^{\prime}}
$$

where $\mathbf{e}_{a}(b \mid \xi)$ is the $\alpha$ component of the vibration eigenvector for the atom $b$ involved in the phonon mode $\xi$ and $D_{\alpha \beta}\left(b b^{\prime} \mid q\right)$ is the component of the dynamical matrix. $\Phi \alpha \beta\left(0 b, /^{\prime} b{ }^{\prime}\right)$ is the real-space force constant between the atom $b$ in the origin unit cell and the atom $b^{\prime}$ in the $l^{\prime}$ th unit cell in the system.

The zeroth-order solution of iterative process is equivalent to the SMRTA, which has been widely adopted for its easier implementation and its explicit relationship with phonon mean free path ${ }^{45,53}$. Details about SMRTA have been introduced in our previous work ${ }^{54}$. For many bulk materials ${ }^{45,53}$, the predictions by SMRTA are close to those from iterative approach. Here, compared with the results from the iterative method, SMRTA leads to almost the same results for $\kappa_{L}$ and relaxation times of $\mathrm{CoSb}_{3}$ and the deviations are generally less than $1 \%$ within the temperature range interested, 
Based on SMRTA, the Normal and Umklapp scattering rates can be obtained, respectively.

Ab initio calculation of IFCs. The harmonic and third-order anharmonic IFCs were obtained from first-principles calculations, which were implemented with the projector augmented wave (PAW $)^{55}$ pseudopotentials, LDA of Perdew and Zunger ${ }^{56}$ and the Perdew-Burke-Ernzerhof $(\mathrm{PBE})^{57}$ form of GGA using the Vienna Ab initio simulation package ${ }^{58}$ (VASP). A conventional unit cell of $\mathrm{CoSb}_{3}$ with 32 atoms was first relaxed with a $11 \times 11 \times 11$ Monkhorst-Pack k sampling grid and a cut-off energy of $400 \mathrm{eV}$. The IFCs were then calculated in the real space using the frozenphonon method $^{59}$. A $2 \times 2 \times 2$ supercell and a conventional unit cell were used for second-order and third-order IFCs calculations, respectively. The static firstprinciples calculations were conducted with a precision as high as $10^{-8} \mathrm{eV}$ for the total energy difference between two self-consistency steps and $4 \times 4 \times 4 \mathbf{k}$-points to obtain the forces on each atom within the perturbed systems. The convergence criterion for the forces on atoms was set to $10^{-6} \mathrm{eV} / \AA$. After the extraction of the harmonic and anharmonic IFCs, the frequency and relaxation time of each mode were calculated by conducting a Fourier transformation with a dense $\mathbf{q}$ mesh (reciprocal space mesh for phonons) scheme $11 \times 11 \times 11$, corresponding to a $11 \times$ $11 \times 11$ supercell in the real space, which has been tested to be enough to yield convergent results in the temperature range from $100 \mathrm{~K}$ to $900 \mathrm{~K}$. Further increase in the mesh scale has little effect on the predicted thermal conductivity values.

1. Uher, C. Skutterudites: Prospective novel thermoelectrics. Recent Trends in Thermoelectric Materials Research I 69, 139-253 (2001).

2. Sales, B., Mandrus, D. \& Williams, R. Filled skutterudite antimonides: A new class of thermoelectric materials. Science 272, 1325-1328 (1996).

3. Koza, M. M. et al. Breakdown of phonon glass paradigm in La- and Ce-filled $\mathrm{Fe}_{4} \mathrm{Sb}_{12}$ skutterudites. Nat. Mater. 7, 805-810 (2008).

4. Kim, H. et al. Structural Order-Disorder Transitions and Phonon Conductivity of Partially Filled Skutterudites. Phys. Rev. Lett. 105, 265901 (2010).

5. Shi, X. et al. Multiple-Filled Skutterudites: High Thermoelectric Figure of Merit through Separately Optimizing Electrical and Thermal Transports. J. Am. Chem. Soc. 133, 7837-7846 (2011).

6. Guo, L., Xu, X., Salvador, J. R. \& Meisner, G. P. Coupled vibrational modes in multiple-filled skutterudites and the effects on lattice thermal conductivity reduction. Appl. Phys. Lett. 102, 111905 (2013).

7. Guo, L., Xu, X., Salvador, J. R. \& Meisner, G. P. Resonant Oscillations in MultipleFilled Skutterudites. J Electron Mater 42, 1978-1981 (2013).

8. Chi, H. et al. Configuring pnicogen rings in skutterudites for low phonon conductivity. Phys. Rev. B 86, 195209 (2012).

9. Liu, W., Zhang, B., Zhao, L. \& Li, J. Improvement of Thermoelectric Performance of $\mathrm{CoSb}_{3-\mathrm{x}} \mathrm{Te}_{\mathrm{x}}$ Skutterudite Compounds by Additional Substitution of IVB-Group Elements for Sb. Chem. Mat. 20, 7526-7531 (2008).

10. Su, X. et al. Microstructure and thermoelectric properties of $\operatorname{CoSb}_{2.75} \mathrm{Ge}_{0.25-\mathrm{x}} \mathrm{Te}_{\mathrm{x}}$ prepared by rapid solidification. Acta Mater. 60, 3536-3544 (2012).

11. Su, X. et al. Structure and Transport Properties of Double-Doped $\mathrm{CoSb}_{2.75} \mathrm{Ge}_{0.25-\mathrm{x}} \mathrm{Te}_{\mathrm{x}}(\mathrm{x}=0.125-0.20)$ with in Situ Nanostructure. Chem. Mat. 23, 2948-2955 (2011).

12. Toprak, M. et al. The impact of nanostructuring on the thermal conductivity of thermoelectric $\mathrm{CoSb}_{3}$. Adv. Funct. Mater. 14, 1189-1196 (2004).

13. Qiu, Y. et al. Charge-Compensated Compound Defects in Ga-containing Thermoelectric Skutterudites. Adv. Funct. Mater. 23, 3194-3203 (2013).

14. Nolas, G., Morelli, D. \& Tritt, T. Skutterudites: A phonon-glass-electron crystal approach to advanced thermoelectric energy conversion applications. Annu. Rev. Mater. Sci. 29, 89-116 (1999).

15. Lutz, H. \& Kliche, G. Far-Infrared Reflection Spectra, Optical and DielectricConstants, Effective Charges, and Lattice-Dynamics of the Skutterudites $\mathrm{CoP}_{3}$, $\mathrm{CoAs}_{3}$, and $\mathrm{CoSb}_{3}$. Phys. Status Solidi B-Basic Res. 112, 549-557 (1982).

16. Nolas, G., Slack, G., Caillat, T. \& Meisner, G. Raman scattering study of antimonybased skutterudites. J. Appl. Phys. 79, 2622-2626 (1996).

17. Nolas, G. \& Kendziora, C. Raman spectroscopy investigation of lanthanide-filled and unfilled skutterudites. Phys. Rev. B 59, 6189-6192 (1999).

18. Rotter, M. et al. Lattice dynamics of skutterudites: Inelastic X-ray scattering on CoSb $_{3}$. Phys. Rev. B 77, 144301 (2008).

19. Ghosez, P. \& Veithen, M. First-principles study of filled and unfilled antimony skutterudites. J. Phys. -Condes. Matter 19, 096002 (2007).

20. Huang, B. \& Kaviany, M. Filler-reduced phonon conductivity of thermoelectric skutterudites: Ab initio calculations and molecular dynamics simulations. Acta Mater. 58, 4516-4526 (2010).

21. Feldman, J. \& Singh, D. Lattice dynamics of skutterudites: First-principles and model calculations for $\mathrm{CoSb}_{3}$. Phys. Rev. B 53, 6273-6282 (1996).

22. Slack, G. \& Tsoukala, V. Some Properties of Semiconducting IrSb 3 . J. Appl. Phys. 76, 1665-1671 (1994).

23. Li, W. \& Mingo, N. Thermal conductivity of fully filled skutterudites: Role of the filler. Phys.Rev.B 89, 184304 (2014).

24. Schmidt, T., Kliche, G. \& Lutz, H. Structure Refinement of Skutterudite-Type Cobalt Triantimonide, $\mathrm{CoSb}_{3}$. Acta Crystallogr. Sect. C-Cryst. Struct. Commun $\mathbf{4 3}$, 1678-1679 (1987).

25. Mahan, G. Good thermoelectrics. Solid State Phys. 51, 81-157 (1998).
26. Caillat, T., Borshchevsky, A. \& Fleurial, J. Properties of single crystalline semiconducting $\mathrm{CoSb}_{3}$. J. Appl. Phys. 80, 4442-4449 (1996).

27. Keppens, V. et al. Localized vibrational modes in metallic solids. Nature $\mathbf{3 9 5}$, 876-878 (1998).

28. Zhang, J., Zhao, G. \& Liang, X. First-Principle Studies of Phonons III-N Compound Semiconductors in Wurtzite Structure. Int. J. Appl. Phys. 3, 227 (2013).

29. Morelli, D. T. et al. Low-Temperature Transport-Properties of P-Type $\mathrm{CoSb}_{3}$. Phys. Rev. B 51, 9622-9628 (1995).

30. Lee, S. et al. Resonant bonding leads to low lattice thermal conductivity. Nat. Commun. 5, 3525 (2014).

31. Esfarjani, K., Chen, G. \& Stokes, H. T. Heat transport in silicon from firstprinciples calculations. Phys. Rev. B 84, 085204 (2011).

32. Gusev, A., Gibin, A., Morozkin, O., Gavva, V. \& Mitin, A. Thermal conductivity of ${ }^{28} \mathrm{Si}$ from 80 to $300 \mathrm{~K}$. Inorg. Mater. 38, 1100-1102 (2002).

33. Dove, M. T. Introduction to lattice dynamics (Cambridge university press, 1993).

34. Guo, R. \& Huang, B. Thermal transport in nanoporous Si: Anisotropy and junction effects. Int. J. Heat Mass Transfer 77, 131-139 (2014).

35. Callaway, J. Model for lattice thermal conductivity at low temperatures. Physical Review 113, 1046 (1959).

36. Ward, A. \& Broido, D. A. Intrinsic phonon relaxation times from first-principles studies of the thermal conductivities of Si and Ge. Phys. Rev. B 81, 085205 (2010).

37. Tian, Z., Esfarjani, K., Shiomi, J., Henry, A. S. \& Chen, G. On the importance of optical phonons to thermal conductivity in nanostructures. Appl. Phys. Lett. 99, 053122 (2011).

38. Tian, Z. et al. Phonon conduction in $\mathrm{PbSe}, \mathrm{PbTe}$, and $\mathrm{PbTe}_{1-\mathrm{x}} \mathrm{Se}_{\mathrm{x}}$ from firstprinciples calculations. Phys. Rev. B 85, 184303 (2012).

39. Nolas, G., Cohn, J. \& Slack, G. Effect of partial void filling on the lattice thermal conductivity of skudderudites. Phys. Rev. B 58, 164-170 (1998).

40. Zhou, Z., Uher, C., Jewell, A. \& Caillat, T. Influence of point-defect scattering on the lattice thermal conductivity of solid solution $\mathrm{Co}\left(\mathrm{Sb}_{1-\mathrm{x}} \mathrm{As}_{\mathrm{x}}\right)_{3}$. Phys. Rev. B 71, 235209 (2005).

41. Abeles, B. Lattice thermal conductivity of disordered semiconductor alloys at high temperatures. Physical Review 131, 1906 (1963).

42. Broido, D. A., Malorny, M., Birner, G., Mingo, N. \& Stewart, D. A. Intrinsic lattice thermal conductivity of semiconductors from first principles. Appl. Phys. Lett. 91, 231922 (2007).

43. Li, W., Carrete, J. \& Mingo, N. Thermal conductivity and phonon linewidths of monolayer $\mathrm{MoS}_{2}$ from first principles. Appl. Phys. Lett. 103, 253103 (2013).

44. Xie, H., Hu, M. \& Bao, H. Thermal conductivity of silicene from first-principles. Appl. Phys. Lett. 104, 131906 (2014).

45. Ward, A., Broido, D. A., Stewart, D. A. \& Deinzer, G. Ab initio theory of the lattice thermal conductivity in diamond. Phys. Rev. B 80, 125203 (2009).

46. Shiomi, J., Esfarjani, K. \& Chen, G. Thermal conductivity of half-Heusler compounds from first-principles calculations. Phys. Rev. B 84, 104302 (2011).

47. Srivastava, G. P. The physics of phonons (CRC Press, 1990).

48. Liao, B., Lee, S., Esfarjani, K. \& Chen, G. First-principles study of thermal transport in $\mathrm{FeSb}_{2}$. Phys. Rev. B 89, 035108 (2014).

49. Omini, M. \& Sparavigna, A. Beyond the isotropic-model approximation in the theory of thermal conductivity. Phys. Rev. B 53, 9064-9073 (1996).

50. Broido, D., Ward, A. \& Mingo, N. Lattice thermal conductivity of silicon from empirical interatomic potentials. Phys. Rev. B 72, 014308 (2005).

51. Feng, T. \& Ruan, X. Prediction of Spectral Phonon Mean Free Path and Thermal Conductivity with Applications to Thermoelectrics and Thermal Management: A Review. J. Nanomater., 206370 (2014).

52. Togo, A., Oba, F. \& Tanaka, I. First-principles calculations of the ferroelastic transition between rutile-type and $\mathrm{CaCl}_{2}$-type $\mathrm{SiO}_{2}$ at high pressures. Phys. Rev. B 78, 134106 (2008).

53. Li, W., Lindsay, L., Broido, D. A., Stewart, D. A. \& Mingo, N. Thermal conductivity of bulk and nanowire $\mathrm{Mg}_{2} \mathrm{Si}_{\mathrm{x}} \mathrm{Sn}_{1-\mathrm{x}}$ alloys from first principles. Phys. Rev. B 86, 174307 (2012).

54. Wang, X. \& Huang, B. Computational Study of In-Plane Phonon Transport in Si Thin Films. Sci Rep 4, 6399 (2014).

55. Blochl, P. Projector Augmented-Wave Method. Phys. Rev. B 50, 17953-17979 (1994).

56. Perdew, J. \& Zunger, A. Self-Interaction Correction to Density-Functional Approximations for Many-Electron Systems. Phys. Rev. B 23, 5048-5079 (1981).

57. Perdew, J., Burke, K. \& Ernzerhof, M. Generalized gradient approximation made simple. Phys. Rev. Lett. 77, 3865-3868 (1996).

58. Kresse, G. \& Furthmuller, J. Efficient iterative schemes for ab initio total-energy calculations using a plane-wave basis set. Phys. Rev. B 54, 11169-11186 (1996).

59. Vanderbilt, D., Taole, S. \& Narasimhan, S. Anharmonic Elastic and Phonon Properties of Si. Phys. Rev. B 40, 5657-5668 (1989).

\section{Acknowledgments}

We are thankful for the financial support from the Hong Kong General Research Fund (Grant Nos. 613211 and 623212) and National Natural Science Foundation of China (Grant No. 51376154). 


\section{Author contributions}

R.G. carried out the ab-initio calculations and data analysis and prepared all the figures. X.W. developed the code. R.G. and B.H. wrote the manuscript. All authors have reviewed, discussed and approved the results and conclusions of this article.

\section{Additional information}

Supplementary information accompanies this paper at http://www.nature.com/ scientificreports
Competing financial interests: The authors declare no competing financial interests. How to cite this article: Guo, R., Wang, X. \& Huang, B. Thermal conductivity of skutterudite $\mathrm{CoSb}_{3}$ from first principles: Substitution and nanoengineering effects. Sci. Rep. 5, 7806; DOI:10.1038/srep07806 (2015).

This work is licensed under a Creative Commons Attribution 4.0 International License. The images or other third party material in this article are included in the article's Creative Commons license, unless indicated otherwise in the credit line; if the material is not included under the Creative Commons license, users will need to obtain permission from the license holder in order to reproduce the material. To view a copy of this license, visit http://creativecommons.org/licenses/by/4.0/ 